\title{
ERRATA
}

\section{Functional annotation of chemical libraries across diverse biological processes}

Jeff S Piotrowski, Sheena C Li, Raamesh Deshpande, Scott W Simpkins, Justin Nelson, Yoko Yashiroda, Jacqueline M Barber, Hamid Safizadeh, Erin Wilson, Hiroki Okada, Abraham A Gebre, Karen Kubo, Nikko P Torres, Marissa A LeBlanc, Kerry Andrusiak, Reika Okamoto, Mami Yoshimura, Eva DeRango-Adem, Jolanda van Leeuwen, Katsuhiko Shirahige, Anastasia Baryshnikova, Grant W Brown, Hiroyuki Hirano, Michael Costanzo, Brenda Andrews, Yoshikazu Ohya, Hiroyuki Osada, Minoru Yoshida, Chad L Myers \& Charles Boone

Nat. Chem. Biol. 13, 982-993 (2017); corrected online 7 August 2017; corrected after print 4 October 2017

In the version of this article initially published, the artemisinin structure in Figure $4 \mathrm{~b}$ was shown with incorrect stereochemistry. The error has been corrected in the HTML and PDF versions of the article.

\section{ERRATA}

\section{Functional annotation of chemical libraries across diverse biological processes}

Jeff S Piotrowski, Sheena C Li, Raamesh Deshpande, Scott W Simpkins, Justin Nelson, Yoko Yashiroda, Jacqueline M Barber, Hamid Safizadeh, Erin Wilson, Hiroki Okada, Abraham A Gebre, Karen Kubo, Nikko P Torres, Marissa A LeBlanc, Kerry Andrusiak, Reika Okamoto, Mami Yoshimura, Eva DeRango-Adem, Jolanda van Leeuwen, Katsuhiko Shirahige, Anastasia Baryshnikova, Grant W Brown, Hiroyuki Hirano, Michael Costanzo, Brenda Andrews, Yoshikazu Ohya, Hiroyuki Osada, Minoru Yoshida, Chad L Myers \& Charles Boone

Nat. Chem. Biol. 13, 982-993 (2017); corrected online 7 August 2017

In the version of this article initially published online, there were several typographical errors introducing scientific inaccuracies. In Figure 5a, the compound name NPE1136 was incorrectly written NPD1136. In the Discussion, the number of strains tested, 5,000, was incorrectly given as $~ 4,900$. In the Online Methods, several strain descriptions in the sections "Constructing a genome-wide drug sensitive yeast deletion correction" and "Assessing compound hit rate of sensitized yeast strains" were incorrect or unclear (in particular, the MATa $x x x \Delta:: k a n M X$ yeast strain was indicated as MA Tax $x x \Delta: k a n M X)$; the unit $\mathrm{mL}$ was given in place of the correct $\mu \mathrm{L}$ in several places in the sections "Multi-parameter validation of cell wall targeting compounds" and "Zymolyase sensitivity assay"; references 14 and 15 were cited instead of references 8 and 17 in the section "Comparison with other chemical-genetic data sets section"; and the list of molecular descriptors calculated using PaDEL-Descriptor in the section "Computing molecular descriptors for all screened compounds" should have started with column L, not J. These errors have been corrected in all versions of the article. 